\title{
BOX-BASKET-BALL SYSTEMS
}

\author{
THOMAS LAM, PAVLO PYLYAVSKYY, AND REIHO SAKAMOTO
}

\begin{abstract}
Using the whurl relation of the first two authors, we define a new discrete solitonic system, which we call the box-basket-ball system, generalizing the box-ball system of Takahashi and Satsuma. In box-basket-ball systems balls may be put either into boxes or into baskets. While boxes stay fixed, both balls and baskets get moved during time evolution. Balls and baskets behave as fermionic and bosonic particles respectively. We classify the solitons of this system, and study their scattering.
\end{abstract}

\section{INTRODUCTION}

In 1990 Takahashi-Satsuma [TS, T] introduced a new discrete soliton system called the box-ball system (BBS). Their discovery is an outcome of the effort to find a cellular automaton with solitonic behaviour, which was a rather popular subject in the 1980s, including the filter automata introduced by Park-Steiglitz-Thurston [PST]. A state of the BBS consists of an infinite sequence of boxes where each box can accommodate at most one ball. Then the time evolution rule of the BBS is described by a simple combinatorial rule in terms of the box and ball interpretation of the system.

A striking feature of the BBS is that, despite its simple outlook, it exhibits all characteristic properties of solitonic systems. This remarkable property of the BBS is a sign of deep mathematical structures behind the BBS. In [TTMS], the authors realized the BBS as the ultra-discrete (or tropical) limit of an ordinary soliton system, thereby proving the integrability of the BBS. Another line of the development is the generalization of the original BBS by introducing extra degrees of freedom like spices of balls or capacity of carriers [T, TM]. Such generalizations eventually culminated in the discovery of the connection with Kashiwara's crystal bases theory [K] of quantum affine algebras found by many authors [HHIKTT, FOY]. Combining these two results, the BBS is now understood as both a classical integrable system and a quantum integrable system.

Recently, yet another mathematical structure behind the BBS was revealed. In [KOSTY], the inverse scattering formalism of the BBS is established with the aid of the theory of rigged configurations. Rigged configurations are certain combinatorial objects originally introduced by Kerov-Kirillov-Reshetikhin [KKR] through their study of the Bethe ansatz analysis for quantum spin chains. As an application, the initial value problem of the BBS is solved [KSY, S1] including all generalizations of the BBS in [HHIKTT, FOY].

Nowadays, reversing the direction of these developments, the BBS also helps to develop new mathematical theories. For example, in [S2], the mysterious algorithm for the bijection between rigged configurations and tensor products of crystals of type $A_{n}^{(1)}$ is identified with the energy functions of crystal bases theory via the time evolution operator of the BBS. Another example is that the inverse scattering formalism of the BBS gave a motivation for generalizing rigged configurations to include not only highest weight elements but also arbitrary elements in tensor products of crystals [S3, DS]. Such a generalization of the rigged configurations have also proven to be useful on representation theory side. Indeed, 
in OSS, the rigged configuration gives an interesting insight into the crystal structure of the Kirillov-Reshetikhin crystals of type $D_{n}^{(1)}$ introduced by [S4]. These developments show that the BBS is not only a model in mathematical physics but also gives a source for future mathematical theories.

The aim of the present article is to propose an entirely different generalization of the BBS. As discussed above, the BBS is related with the crystal bases theory. To be more specific, the time evolution operators of the BBS is described by the combinatorial $R$ matrix of the crystal bases theory. In [LP], a generalization of the type $A_{n}^{(1)}$ combinatorial $R$-matrix, which they called the whurl relation, are introduced through the study of networks on a cylinder.

Let us explain the whurl relation in more detail. The whurl relation is parametrized by vertical wires (pointing up) and horizontal wires (pointing right or left) on a cylinder. If all the horizontal wires point to the right, the whurl relation reduces to the original combinatorial $R$-matrices for the symmetric tensor product representation of type $A_{n}^{(1)}$. On the other hand, if some of wires point to the left, the whurl relation becomes a new map satisfying the Yang-Baxter relation. Thus we can expect that the whurl relation will generate another class of quantum integrable systems generalizing the BBS.

Among the whurl relation, the simplest possible non-trivial extension is the three horizontal wires case, where two of the wires point to the right and the other one points to the left (see section 2.3). In the present article, we concentrate only on this three wire case in order to clarify the most fundamental properties of the new system without getting into the technical complexities concerning the general whurl relation. In this way we stay close in spirit to the original Takahashi-Satsuma paper [TS].

The resulting new system, which we coin the box-basket-ball system (BBBS), has a remarkable novel property, namely, it contains two entirely different kinds of particles whereas the BBS and its generalizations have essentially one type of particles (of various internal degrees of freedom). In section [3.1, we provide a combinatorial description of the system in terms of boxes, balls and baskets, generalizing the description for the BBS. Here each box or basket can accommodate at most one ball, whereas baskets can be put more than one on a box. In this sense, we can regard balls as fermionic particles and baskets as bosonic particles, together with mutual interaction between balls and baskets. In section 3.2, we show that this combinatorial description agrees with the definition in terms of the whurl relation. As it turns out, the baskets originate from the wire pointing to the left in the network on the cylinder.

Since the whurl relation satisfies the Yang-Baxter relation, we see that our BBBS is a quantum integrable system (Theorem 4.4). However it is still a non-trivial problem to show that the BBBS is indeed a soliton system. The rest of the paper is devoted to show the solitonic property of the BBBS as described in Theorem 5.8. In order to achieve this goal, we first classify all possible solitary waves that propagate without changing their shapes if there is no scattering (Proposition 4.2). A novel property is the presence of slow solitons. This new kind of solitary waves are caused by a difference in the phase shifts of the scatterings for two types of velocity one solitons as described in Proposition 5.1 and Corollary 5.3, respectively. Finally we give a careful analysis of the general scatterings in Proposition 5.2 which forms the technical heart of the present paper.

We give a comment on related works. In [HI], the authors constructed a supersymmetric extension of the BBS by using the crystal bases for the quantum superalgebra introduced 
by [BKK]. Their system is different from ours since their extension is due to the addition of fermionic particles to the original BBS whereas the BBBS is obtained by adding bosonic particles to the BBS.

Let us say a few words about possible future directions of the present study. Recall that in the original BBS case, the solitonic property of the system is a sign of rich mathematical structures behind the model. In our BBBS case, we also established the solitonic property of the system. Thus it is not unreasonable to expect that the unknown symmetry behind the general whurl relation might have deeper properties and it will be worthy to clarify such underlying symmetry behind our system.

Another possibility we have in mind is for engineering purposes related to transportation problems or traffic flow problems. Since our system possesses obstacles described by baskets, we hope that the integrability of our system might provide some tools for detailed analysis of such problems. An alternative physical model would be waves in shallow water with some sand on the bottom. The sand represents the slow bosonic particles, while the water represents the fast fermionic particles.

\section{Discrete DyNAMiCAL SYSTEMS ARISING FROM WHURL RELATIONS}

2.1. Takahashi-Satsuma box-ball system. In [TS, T] Takahashi and Satsuma defined a discrete dynamical system exhibiting solitonic behaviour. We have a sequence of sites $\left\{S_{i} \mid i \in \mathbb{Z}\right\}$. Each site $S_{i}$ contains one box which is either empty or has a ball inside. We denote the state of $S_{i}$ by a pair of numbers $\left(a_{i}, b_{i}\right)$, where $b_{i}$ is the number of balls in it and $a_{i}$ is the number of extra balls that could fit in. Thus, each site is either in state $(1,0)$, which we call the vacuum state, or in state $(0,1)$, which we call the ball state. The notation is set up so that one could also consider the case of more than one box at a site. We shall however consider only the simple case of one box. We assume that $S_{i}=(1,0)$ for $i \ll 0$ or $i \gg 0$.

Time evolution of the system is as follows. The carrier travels from left $(i \ll 0)$ to right $(i \gg 0)$, having an infinite capacity to carry balls. She performs the following operations at each site $S_{i}$ :

- if $S_{i}=(0,1)$, she picks up this ball, changing the state into the vacuum $S_{i}=(1,0)$;

- if $S_{i}=(1,0)$, and she is carrying at least one ball, she drops one ball, changing the state of the site into $S_{i}=(0,1)$.

In other words, we consider each ball from left to right (that is, starting from $S_{-\infty}$ ) and move the ball to the next available box. Each ball is moved exactly once. This completes the time evolution.

A soliton is a sequence of states which evolves at constant speed with no change in internal structure. A basic soliton is a soliton $A$ which cannot be decomposed into (nontrivial) solitons $A^{\prime}$ and $A^{\prime \prime}$ where $A^{\prime}$ and $A^{\prime \prime}$ are separated by at least as many vacuum states as the speed of $A$.

\section{Theorem 2.1. [TS}

(1) The basic solitons of box-ball system are strings of consecutive balls; the soliton of length $k$ has speed $k$.

(2) When a system consisting of a disjoint union of (suitably separated) basic solitons is allowed to scatter, the outcome is a sequence of basic solitons with the same set of lengths, arranged in non-decreasing order from left to right. 
(3) Starting at any initial state, after a finite amount of time the system separates into basic solitons with non-decreasing length.

One can also describe the action of the carrier as follows. Assume the carrier is in state $(a, b)$ and the site she is going through is in state $(c, d)$. Then the new state of the carrier and the site are given by:

$$
\begin{aligned}
a^{\prime} & =a+\min (b, c)-\min (a, d) \\
b^{\prime} & =b-\min (b, c)+\min (a, d) \\
c^{\prime} & =c-\min (b, c)+\min (a, d) \\
d^{\prime} & =d+\min (b, c)-\min (a, d)
\end{aligned}
$$

It is easy to check that when $a=\infty$ this gives the action of the carrier described above. Thus, one can assume that the carrier is originally in the state $(\infty, 0)$ and acts on each site according to the transformation above.

2.2. Combinatorial $R$-matrix and whurl relations. One can give a crystal base [K] formulation of the box-ball systems [HHIKTT, FOY] (see [TM] for a combinatorial description). The transformation describing the carrier action is a special case of the combinatorial $R$-matrix arising in the theory of crystals. In this context, states of the system are regarded as elements of tensor products of the crystals $\cdots \otimes b_{i-1} \otimes b_{i} \cdots \in$ $\cdots \otimes B_{k_{i-1}} \otimes B_{k_{i}} \otimes B_{k_{i+1}} \otimes \cdots$.

In [LP] certain birational transformations constructed from networks on oriented surfaces were considered. The tropicalization of one of these transformations turns out to be exactly the combinatorial $R$-matrix associated to the box-ball system. More specifically, consider a cylinder with several parallel horizontal wires connecting the components of the boundary, and several closed disjoint loops going around the cylinder. All the loops are oriented in the same direction. The orientations of the horizontal wires are allowed to vary: each is oriented either from left to right or from right to left. A parameter is associated to each vertex of the resulting network. The case of two horizontal wires, both oriented to the right, and two loops is shown in Figure 1.

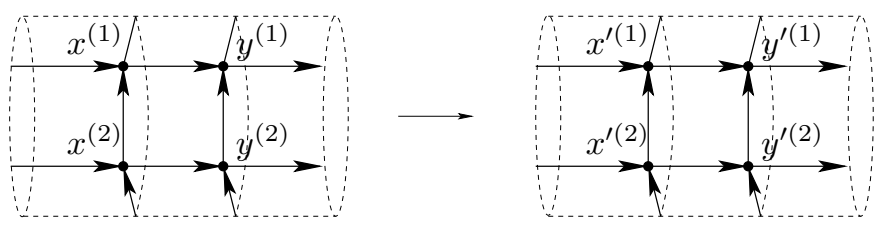

FiguRE 1. An example of the whurl relation.

The loops around the cylinder are called whurls. In [LP] transformations of parameters of adjacent whurls were studied that preserve boundary measurements in the networks. The whurl relation in the case shown in Figure 1 is given by

$$
\begin{aligned}
& x^{\prime(1)}=y^{(1)} \frac{x^{(1)}+y^{(2)}}{y^{(1)}+x^{(2)}} ; \quad x^{\prime(2)}=y^{(2)} \frac{x^{(2)}+y^{(1)}}{y^{(2)}+x^{(1)}} ; \\
& y^{\prime(1)}=x^{(1)} \frac{x^{(2)}+y^{(1)}}{y^{(2)}+x^{(1)}} ; \quad y^{\prime(2)}=x^{(2)} \frac{x^{(1)}+y^{(2)}}{y^{(1)}+x^{(2)}} .
\end{aligned}
$$


Note that with $a=y^{(1)}, b=y^{(2)}, c=x^{(1)}, d=x^{(2)}$, this transformation recovers the piecewise-linear description of the box-ball system carrier, under the tropicalization (also called ultradiscretization $)(+, \times) \mapsto(\min ,+)$. We denote the whurl transformation by $R$.

Theorem 2.2. [LP, Proposition 11.8] When all $n$ horizontal wires have the same orientation, $R$ coincides with the birational version of the combinatorial $R$-matrix of KirillovReshetikhin crystals for symmetric powers of the standard representation of $U_{q}^{\prime}\left(A_{n}^{(1)}\right)$.

In what follows we shall use the following property of $R$. Suppose we are given three adjacent whurls labeled 1,2,3 from left to right. Let $R_{i j}$ be the whurl relation acting on the whurls labeled $i$ and $j$.

Theorem 2.3. [LP, Theorem 6.6] The whurl relation (and thus, its tropicalization) satisfies the Yang-Baxter relation

$$
\left(R_{12} \otimes 1\right) \circ\left(1 \otimes R_{23}\right) \circ\left(R_{12} \otimes 1\right)=\left(1 \otimes R_{23}\right) \circ\left(R_{12} \otimes 1\right) \circ\left(1 \otimes R_{23}\right) .
$$

2.3. The mixed wires case and a box-basket-ball system. The box-ball systems corresponding to whurl relations with all horizontal wires oriented in the same direction have been extensively studied in literatures. In particular, there are generalizations to systems with balls of many different colors, or to systems with boxes with higher capacity. As suggested by the model of networks on surfaces in [LP], one may consider a more general setting of having wires in both directions. The action of the carrier in such systems would be given by tropicalization of the corresponding whurl relations. We make the following general conjecture.

Conjecture 2.4. The discrete dynamical systems arising from general whurl relations exhibit solitonic behaviour.

In this paper we shall consider the case of three horizontal wires, where two are oriented to the right and one to the left, as shown in Figure 2. The whurl relation in this case is

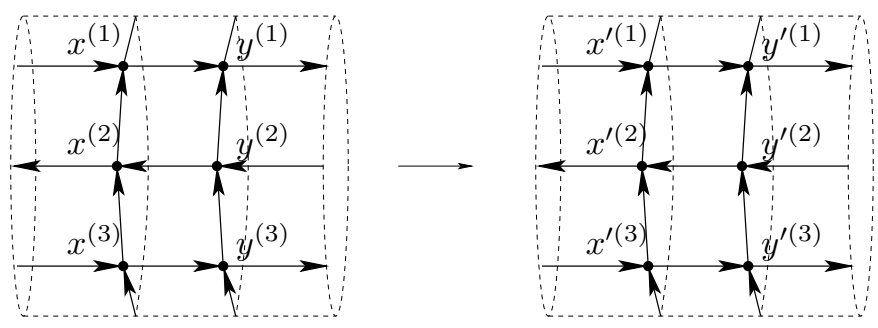

Figure 2. A whurl relation with mixed directions of wires.

given by

$$
\begin{gathered}
x^{(1)}=y^{(1)} \frac{x^{(1)} x^{(2)}+x^{(1)} x^{(3)}+x^{(2)} y^{(3)}}{y^{(2)} x^{(3)}+y^{(1)} x^{(3)}+y^{(1)} x^{(2)}} \quad x^{(2)}=y^{(2)} \frac{x^{(1)} x^{(2)}+x^{(1)} x^{(3)}+x^{(2)} y^{(3)}}{x^{(1)} y^{(2)}+y^{(1)} y^{(3)}+y^{(2)} y^{(3)}} \\
x^{\prime(3)}=y^{(3)} \frac{y^{(1)} x^{(3)}+y^{(1)} x^{(2)}+y^{(2)} x^{(3)}}{x^{(1)} y^{(2)}+y^{(1)} y^{(3)}+y^{(2)} y^{(3)}} \\
y^{\prime(1)}=x^{(1)} \frac{y^{(2)} x^{(3)}+y^{(1)} x^{(3)}+y^{(1)} x^{(2)}}{x^{(1)} x^{(2)}+x^{(1)} x^{(3)}+x^{(2)} y^{(3)}} \quad y^{\prime(2)}=x^{(2)} \frac{x^{(1)} y^{(2)}+y^{(1)} y^{(3)}+y^{(2)} y^{(3)}}{x^{(1)} x^{(2)}+x^{(1)} x^{(3)}+x^{(2)} y^{(3)}}
\end{gathered}
$$




$$
y^{\prime(3)}=x^{(3)} \frac{x^{(1)} y^{(2)}+y^{(1)} y^{(3)}+y^{(2)} y^{(3)}}{y^{(1)} x^{(3)}+y^{(1)} x^{(2)}+y^{(2)} x^{(3)}}
$$

In the next section we shall build a discrete dynamical system based on the tropicalization of this transformation, that we call the box-basket-ball system.

\section{BOX-BASKET-BALL SYSTEM}

3.1. Combinatorial description. We have a sequence of sites $\left\{S_{i} \mid i \in \mathbb{Z}\right\}$. Each site $S_{i}$ contains one box, a number $b_{i}$ of baskets, and a number $c_{i}$ of balls. Each ball must occupy a box or a basket, so that $c_{i} \leq b_{i}+1$. We define $a_{i}=b_{i}-c_{i}+1$, the number of extra balls that can fit. To denote the state of $S_{i}$, we will often use the vector $\left(a_{i}, b_{i}, c_{i}\right)$. The vacuum state is $(1,0,0)$ - one empty box and no baskets or balls. We assume that $S_{i}=(1,0,0)$ for $i \ll 0$ or $i \gg 0$.

Time evolution of the system is as follows. Before beginning, we assume that a ball is always placed in a box if that is possible (before being placed in a basket). This does not change the state $\left(a_{i}, b_{i}, c_{i}\right)$. First, we move every empty basket to the right one step (that is, from $S_{i}$ to $S_{i+1}$ ). Full baskets are not moved. Second, we consider each ball from left to right (that is, starting from $S_{-\infty}$ ) and move the ball to the next available box or basket. Each ball is moved exactly once. This completes the time evolution.

3.2. Piecewise linear description via carrier. The time evolution can also be achieved via a carrier $C$, which we think of as starting from the left, and initially carrying infinitely many boxes, no baskets, and no balls. The interaction $\left(C, S_{i}\right) \mapsto\left(S_{i}^{\prime}, C^{\prime}\right)$ of the carrier $C=(a=\infty, b, c)$ with a site $S_{i}=(d, e, f)$ is given by

$$
\begin{aligned}
& d^{\prime}=d+b+f-\min (e+c, d+c, d+b) \\
& e^{\prime}=e+b-\min (d, e) \\
& f^{\prime}=\min (e+c, d+c, d+b)-\min (d, e)
\end{aligned}
$$

where $S_{i}^{\prime}=\left(d^{\prime}, e^{\prime}, f^{\prime}\right)$. Balls and baskets are preserved, so that the resulting carrier $C^{\prime}$ is given by

$$
\begin{aligned}
& a^{\prime}=\infty \\
& b^{\prime}=\min (d, e) \\
& c^{\prime}=c+f+\min (d, e)-\min (e+c, d+c, d+b) .
\end{aligned}
$$

It is convenient to express the interaction of the carrier with a site as the vertex diagram:

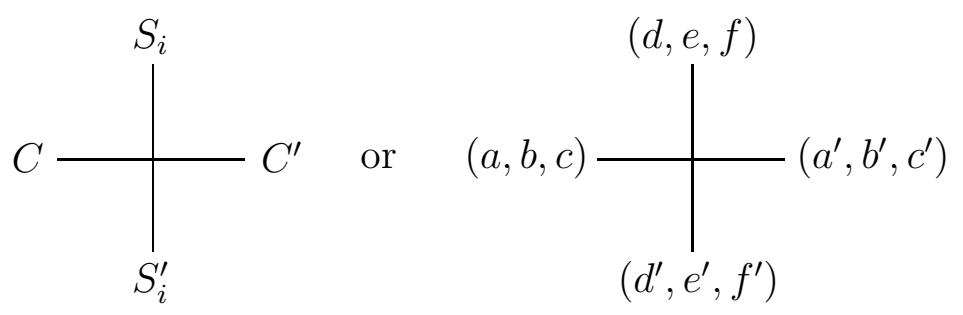

Proposition 3.1. The action of the carrier can be described as follows:

- she picks up all the empty baskets at the site, and drops the baskets she was carrying before;

- then she drops as many of the balls she is carrying as she can, and picks up all the balls that were there before she dropped new ones. 
Proof. Initially there are $\min (d, e)$ empty baskets at the site, which implies the correctness of the rule for baskets. After the baskets are already taken care of, there are $d-\min (d, e)$ empty boxes and $b$ empty baskets at the site, and the correctness of the rule follows from the identity

$$
\min (e+c, d+c, d+b)-\min (d, e)=\min (c, d+b-\min (d, e))
$$

Proposition 3.2. The two descriptions of the time evolution are equivalent.

Proof. The stated piecewise-linear transformation can be factorized as the composition of

$$
\begin{aligned}
& d^{\prime \prime}=d-\min (d, e)+b \\
& e^{\prime \prime}=e-\min (d, e)+b \\
& f^{\prime \prime}=f
\end{aligned}
$$

and

$$
\begin{aligned}
& d^{\prime}=d^{\prime \prime}+f^{\prime \prime}-\min \left(c, d^{\prime \prime}\right) \\
& e^{\prime}=e^{\prime \prime} \\
& f^{\prime}=\min \left(c, d^{\prime \prime}\right)
\end{aligned}
$$

Indeed, as remarked above

$$
\min \left(c, d^{\prime \prime}\right)=\min (e+c, d+c, d+b)-\min (d, e),
$$

and one needs only to plug in the expression for $d^{\prime \prime}, e^{\prime \prime}, f^{\prime \prime}$ into the formulas for $d^{\prime}, e^{\prime}, f^{\prime}$.

The two components of the piecewise-linear time evolution can be carried out independently of each other. That is, one can first let the carrier go through all sites doing only the first transformation, and then let her go through all sites doing only the second transformation. Indeed, the first transformation does not depend on and does not influence the resulting number of balls she carries, while the second transformation does not depend on and does not influence the number of baskets she carries. However, viewed independently from each other, it is clear that the two transformations accomplish exactly the combinatorial description of the time evolution.

Theorem 3.3. The action of the carrier in the box-basket-ball system is a tropicalization of the whurl relation given in Section 2.3.

Proof. Direct computation, plugging in $x^{(1)}=a=\infty$ into the tropicalization of the whurl relation.

3.3. Projection to box-ball system. The box-ball system naturally embeds into the box-basket-ball system, by considering states with no baskets. It turns out that one can also project the box-basket-ball system onto the box-ball system as follows. Take a state of box-basket-ball system at time $t$. Assume we have a site in a state $S=(a, b, c)$, where $a+c-b=1$. Turn this site into a sequence of $b+1$ sites in the usual box-ball system, and fill the first $c$ of them with balls. Do this for every site of the original state, creating a state of the box-ball system. We call the operation the unbasketing of the original state; if the original state does not contain baskets, then unbasketing does not do anything. Note however that there is no canonical way to reverse the unbasketing procedure. 
Theorem 3.4. The time evolution commutes with unbasketing. In other words, applying first the time evolution in the box-basket-ball system and then unbasketing gives the same result as first applying unbasketing and then applying the time evolution in the box-ball system.

Proof. The action of the carrier in the box-basket-ball system can be equivalently described the following way:

- consider the empty baskets standing at the end of the unbasketing of a site $S_{i}$ as the beginning of the unbasketing of $S_{i+1}$;

- move the balls one by one left to right to the first unoccupied position;

- rearrange boxes and baskets without moving balls so that we get the correct unbasketing of the new state.

If we ignore the partitioning into sites in box-basket-ball system, as well as stop distinguishing between baskets and boxes, we are left only with the second step which is exactly the time evolution of the box-ball system.

Example 3.5. Start with the state

$$
\ldots,(1,2,2),(2,4,3),(1,2,2), \ldots,
$$

where the sites not shown are vacuum. Its unbasketing is $\ldots 1,1,0,1,1,1,0,0,1,1, \ldots$ where 1 denotes a ball and 0 denotes a vacuum state, and the sites not shown are vacuum states. If we apply the time evolution to the original state we obtain

$$
\ldots,(2,1,0),(3,3,1),(2,3,2),(0,1,2),(0,0,1),(0,0,1) \ldots,
$$

with unbasketing ..., 1, 0, 0, $0,1,1,0,0,1,1,1,1, \ldots$. It is easily checked that this unbasketing is obtained from the original one by the time evolution of box-ball system.

\section{Solitons}

\subsection{Classification. Denote}

$$
\begin{aligned}
V & =(1,0,0) \\
F & =(0,0,1) \\
B_{a} & =(a+1, a, 0), \quad a \geq 1 \\
U_{a} & =(a, a, 1), \quad a \geq 1 .
\end{aligned}
$$

Example 4.1. Let us depict a box by $\bigsqcup$, a ball by $\bigcirc$, and a basket by . Then the above elements are depicted as follows:

$$
V=\bigsqcup, \quad F=\emptyset, \quad B_{2}=\bigsqcup, \quad U_{2}=\emptyset .
$$

A soliton is a sequence of states which evolves at constant speed with no change in internal structure. A basic soliton is a soliton $A$ which cannot be decomposed into (nontrivial) solitons $A^{\prime}$ and $A^{\prime \prime}$ where $A^{\prime}$ and $A^{\prime \prime}$ are separated by at least as many vacuum states as the speed of $A$.

Proposition 4.2. The basic solitons are

(1) $F_{k}:=\overbrace{F F \cdots F}^{k}$ of speed equal to the length, which we call a fast soliton, 
(2) any string of $F, B, U$ which does not contain the consecutive subsequence $F F$ or $F U$, of speed 1, which we call a slow soliton.

Proof. Any soliton would remain a soliton after projecting to box-ball system via unbasketing. Note that no basket can move with speed greater than one. Thus, if our soliton projects to a soliton of speed greater than one, it has to contain no baskets. A state containing no baskets projects to itself in the box-ball system. Thus by Theorem 2.1 any such soliton would be one of the $F_{k}, k \geq 2$.

Assume now that the soliton has speed one. Then it projects to a collection of disjoint solitons consisting of one ball. Any positioning of boxes with at most one ball at each site has to be a combination of $F, B, U$ (we can assume there are no $V$-s since we are interested in basic solitons of speed one). Since the unbasketting should not contain two consecutive balls, the sequence should avoid $F F$ or $F U$ subsequences.

It remains to argue that any such string of $F, B, U$ is indeed a soliton of speed one. This follows from the carrier description of box-basket-ball system. We argue that carrier always carries at most one ball to the next site, and always carries all the baskets from the previous site. Indeed, the only way it could fail is if she is carrying a ball into a site and this site has already one ball in it. However, since subsequences $F F$ and $F U$ are avoided, she will also be carrying at least one basket, and thus would have where to put the ball.

4.2. Slower time evolution. Consider the time evolution $T_{\ell}$ with carrier $u_{\ell}:=(\ell, 0,0)$ with $1<\ell<\infty$. The interaction $\left(C, S_{i}\right) \mapsto\left(S_{i}^{\prime}, C^{\prime}\right)$ of the carrier $C=(a, b, c)$ with a site $S_{i}=(d, e, f)$ is given by

$$
\begin{aligned}
& d^{\prime}=d+\min (a+b, a+c, b+f)-\min (e+c, d+c, d+b) \\
& e^{\prime}=e+\min (a+b, a+c, b+f)-\min (a+e, d+f, e+f) \\
& f^{\prime}=f+\min (e+c, d+c, d+b)-\min (a+e, d+f, e+f)
\end{aligned}
$$

where $S_{i}^{\prime}=\left(d^{\prime}, e^{\prime}, f^{\prime}\right)$. Balls and baskets are preserved, so that the resulting carrier $C^{\prime}$ is given by

$$
\begin{aligned}
& a^{\prime}=a-\min (a+b, a+c, b+f)+\min (e+c, d+c, d+b) \\
& b^{\prime}=b-\min (a+b, a+c, b+f)+\min (a+e, d+f, e+f) \\
& c^{\prime}=c-\min (e+c, d+c, d+b)+\min (a+e, d+f, e+f)
\end{aligned}
$$

Note that the relations $a^{\prime}-b^{\prime}+c^{\prime}=a-b+c$ and $d^{\prime}-e^{\prime}+f^{\prime}=d-e+f$ hold. Thus the capacities of sites and carriers are preserved under the time evolutions. This is exactly the tropicalization of whurl relations in Section 2.3.

Proposition 4.3. The slower time evolution $T_{\ell}$ of solitons described above is as follows.

(1) the soliton $F_{k}$ moves with speed $\min (\ell, k)$;

(2) the solitons of speed one evolve exactly the same way as before.

Proof. The first part is known [FOY], since in that case box-basket-ball system is indistinguishable from the box-ball system.

For the second part, observe that while acting on a speed one soliton the carrier never has to carry more than one ball at a time. Thus it does not matter what her capacity is as it is at least 1 .

Related to quantum integrability, our system possesses the following property. 
Theorem 4.4. The time evolution operators $\left\{T_{\ell}\right\}_{\ell \geq 1}$ commute with each other.

For the proof, we prepare the following lemma.

Lemma 4.5. Let us consider a path $S=S_{1} \otimes S_{2} \otimes \cdots \otimes S_{m+n}$ where $S_{j}=V$ for $m \leq \forall j \leq m+n$. If $n$ is sufficiently large, we have $u_{l} \otimes S \simeq S^{\prime} \otimes u_{l}$ under the isomorphism $R$.

Proof. Let us consider

$$
(a, b, c) \otimes(1,0,0) \simeq\left(d^{\prime}, e^{\prime}, f^{\prime}\right) \otimes\left(a^{\prime}, b^{\prime}, c^{\prime}\right)
$$

under the whurl relation. Recall that we have $a+c=b+l$ since we start from $u_{l}$. Thus we have $a+c>b$, while $a+b \geq b$. From this we have

$$
b^{\prime}=b-\min (a+b, a+c, b)+\min (a, 1,0)=b-b=0 .
$$

Similarly, if $c>0$, we have

$$
c^{\prime}=c-\min (c, 1+c, 1+b)+\min (a, 1,0)=c-\min (c, b+1)<c .
$$

Since $c$ is finite, if we consider the above isomorphism repeatedly, we will finally obtain $c^{\prime}=0$. In this case, we have $\left(a^{\prime}, b^{\prime}, c^{\prime}\right)=(l, 0,0)$, which proves the lemma.

Proof of Theorem 4.4. Since $S_{i}=V$ for all $|i| \gg 1$, we can choose $m, n$ such that $S=$ $S_{m} \otimes S_{m+1} \otimes \cdots \otimes S_{n}$ satisfies the above lemma as well as $S_{i}=V$ for all $i<m$ and $n<i$. We shall show $T_{k} T_{l}(S)=T_{l} T_{k}(S)$. Apply the Yang-Baxter relation (Theorem 2.3) repeatedly to $u_{k} \otimes u_{l} \otimes S$ as follows:
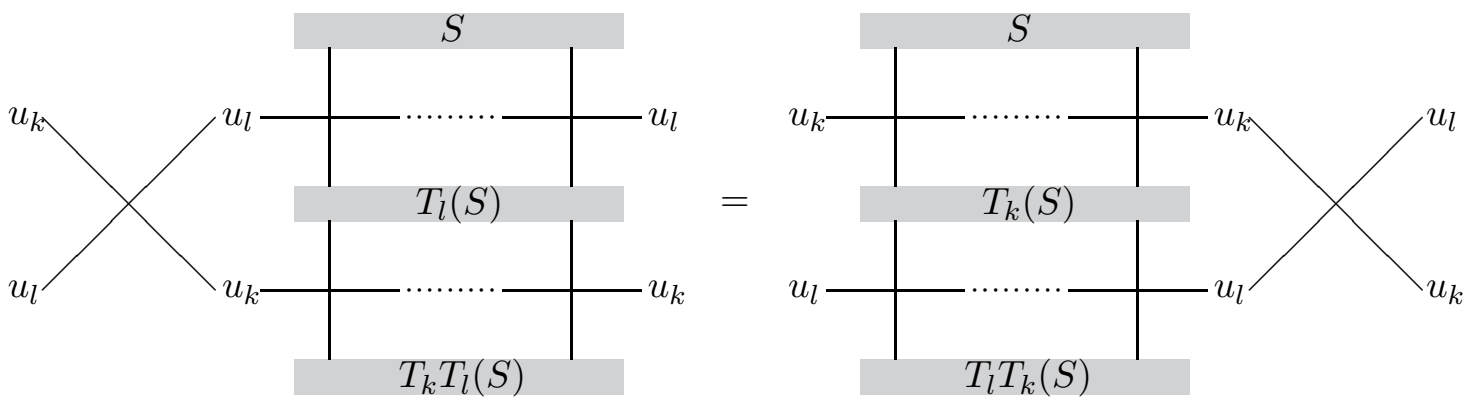

Comparing both sides we obtain $T_{k} T_{l}(S)=T_{l} T_{k}(S)$.

In the case of the original box-ball systems, it is known that the family of mutually commutative time evolutions gives complete information on the action-angle variables of the dynamics $\mathrm{S2}$.

\section{SCATtering}

5.1. Some definitions. We begin by introducing some notation that will be used in the rest of the paper. The following two basic solitons we shall call pure solitons:

$$
\begin{aligned}
& F_{k}=\overbrace{F F \cdots F}^{k}, \\
& B_{a_{1}, a_{2}, \cdots, a_{r}}:=B_{a_{1}} B_{a_{2}} \cdots B_{a_{r}},
\end{aligned}
$$

and the remaining speed one basic solitons of Proposition 4.2 (2) are called composite solitons. 
Let us introduce some notation for our speed 1 basic soliton. The balls in the soliton we shall call slow balls. If there is a ball in the tail (leftmost site) of a soliton, then we call it the initial slow ball. The condition that the soliton does not contain $F F$ or $F U$ is equivalent to the condition that every non-initial slow ball has a basket in the site immediately behind (towards the tail) of it. Let us begin from the tail of the soliton and number all the baskets with $1,2, \ldots, b$. Within the same site, we number from lower baskets to upper baskets. If there is a non-initial slow ball in site $S_{i}$, then we shall call the basket with the highest number in site $S_{i-1}$ special. The $m$-th non-initial slow ball in the soliton and the $m$-th special basket in the soliton are considered paired.

The balls in our speed $k$ soliton are called fast balls.

5.2. Phase shift of scatterings. Suppose $A$ and $A^{\prime}$ are basic solitons, such that after scattering one obtains again the solitons $A^{\prime}$ and $A$ (but now in the reverse order):

$$
p=\cdots A \cdots \cdots A^{\prime} \cdots \longrightarrow T_{\ell}^{N}(p)=\cdots A^{\prime} \cdots \cdots A \cdots
$$

for sufficiently large $N$. Here, "...A..$"$ is the abbreviation for the state $\cdots V \otimes V \otimes A \otimes$ $V \otimes V \otimes \cdots$. Let $v$ and $v^{\prime}$ be the velocities of the free propagations of $A$ and $A^{\prime}$ under the time evolution $T_{\ell}$. Define the phase shifts of $A$ and $A^{\prime}$ before the scattering to be 0 . Let the position of the leftmost letter of $A$ of $p$ be 0 . Then the position of the leftmost letter of $A$ of $T_{\ell}^{N}(p)$ can be expressed as $N v+\delta$. Similarly we define $\delta^{\prime}$ for $A^{\prime}$. We call these $\delta, \delta^{\prime}$ as phase shifts and describe the above scattering as follows:

$$
A[0] * A^{\prime}[0] \longrightarrow A^{\prime}\left[\delta^{\prime}\right] * A[\delta] .
$$

If internal degree of freedom changes after the scattering, we define the phase shift for each ball or basket in the similar way as above by comparing with the corresponding elements before scattering.

5.3. Scattering of fast solitons. The following result for scattering in the box-ball system is well known.

Proposition 5.1. For any time evolution $T_{\ell}(\ell \geq 2)$, the two body scatterings between the fast solitons is as follows $(m>n)$ :

$$
F_{m}[0] * F_{n}[0] \longrightarrow F_{n}[-2 n] * F_{m}[2 n]
$$

5.4. Scattering of fast soliton and slow soliton. The general form of such scattering is as follows:

Proposition 5.2. For any time evolution $T_{\ell}(\ell \geq 2)$, the two body scatterings between the fast soliton $F_{m}: m>1$ and a basic slow soliton $A$ is given by:

$$
F_{m}[0] * A \longrightarrow A^{\prime} * F_{m}[2 b-a],
$$

where $b$ is the total number of balls in $A$, and $a$ is the total number of baskets in $A$, and $A^{\prime}$ is obtained from $A$ as follows:

(1) All non-special baskets, and non-initial slow balls are slowed by 1.

(2) All special baskets are not phase-shifted.

(3) The initial slow ball (if any) is slowed by 2.

Note that because of (3), $A^{\prime}$ may no longer be a basic soliton. 


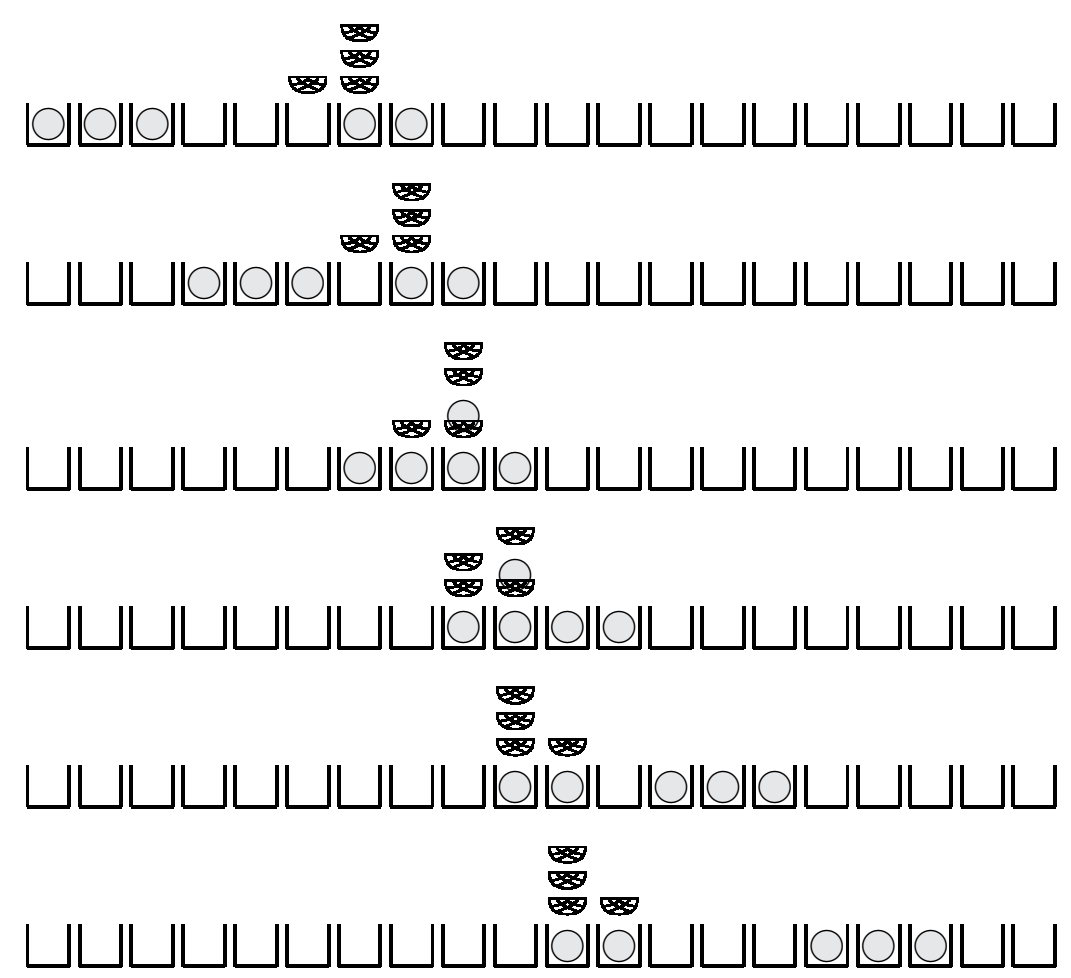

Figure 3. Scattering of $F_{3}$ and $B_{1} U_{3} F$ under $T_{\infty}$.

Proof. For $T_{\infty}$, this result is established in Section 6. To show that the result of scattering using $T_{\ell}$ does not depend on $\ell$, we use the commutativity of the time evolutions (Theorem 4.4). If $p$ denotes the initial state then we have $\left(T_{\ell}\right)^{M}(p)=\left(T_{\infty}\right)^{-N}\left(T_{\ell}\right)^{M}\left(T_{\infty}\right)^{N}(p)$ where $N$ is chosen to be very large and $M$ is chosen even larger. Suppose the scattering for $T_{\infty}$ is known, so that $\left(T_{\infty}\right)^{N}(p)$ is the disjoint union of basic solitons as described in the proposition. Then $\left(T_{\ell}\right)^{M}\left(T_{\infty}\right)^{N}(p)$ consists of the same set of solitions evolving freely at slow $(\leq \ell)$ speed. If $M \gg N$, then $\left(T_{\infty}\right)^{-N}\left(T_{\ell}\right)^{M}\left(T_{\infty}\right)^{N}(p)$ will still consist of the same set of solitions with no (reverse) scattering. Thus the scattering of $T_{\ell}$ and $T_{\infty}$ are the same.

Corollary 5.3. Let a be the total number of baskets in $B_{a_{1}, a_{2}, \cdots, a_{r}}$. Then we have

$$
F_{m}[0] * B_{a_{1}, a_{2}, \cdots, a_{r}}[0] \longrightarrow B_{a_{1}, a_{2}, \cdots, a_{r}}[-1] * F_{m}[-a]
$$

under any time evolution $T_{\ell}(\ell \geq 2)$.

Example 5.4. Consider the scattering of $F_{3}$ with $B_{1} U_{3} F$. Under $T_{\infty}$, it is displayed in Figure 3 and under $T_{2}$ it is displayed in Figure 4 .

Any basic soliton of speed one can be uniquely cut into the chunks of the following kinds:

$$
\begin{aligned}
& B_{a} U_{b_{1}} \ldots U_{b_{r}} Z, \quad B_{a} U_{b_{1}} \ldots U_{b_{r}} F Z, \quad V U_{b_{1}} \ldots U_{b_{r}} Z, \quad V U_{b_{1}} \ldots U_{b_{r}} F Z, \\
& V F Z, \quad B_{a_{1}} \ldots B_{a_{r}} Z .
\end{aligned}
$$

Here $Z$ denotes either $B$ or $V$, though we regard that $Z$ is not a member of the chunk before $Z$. 

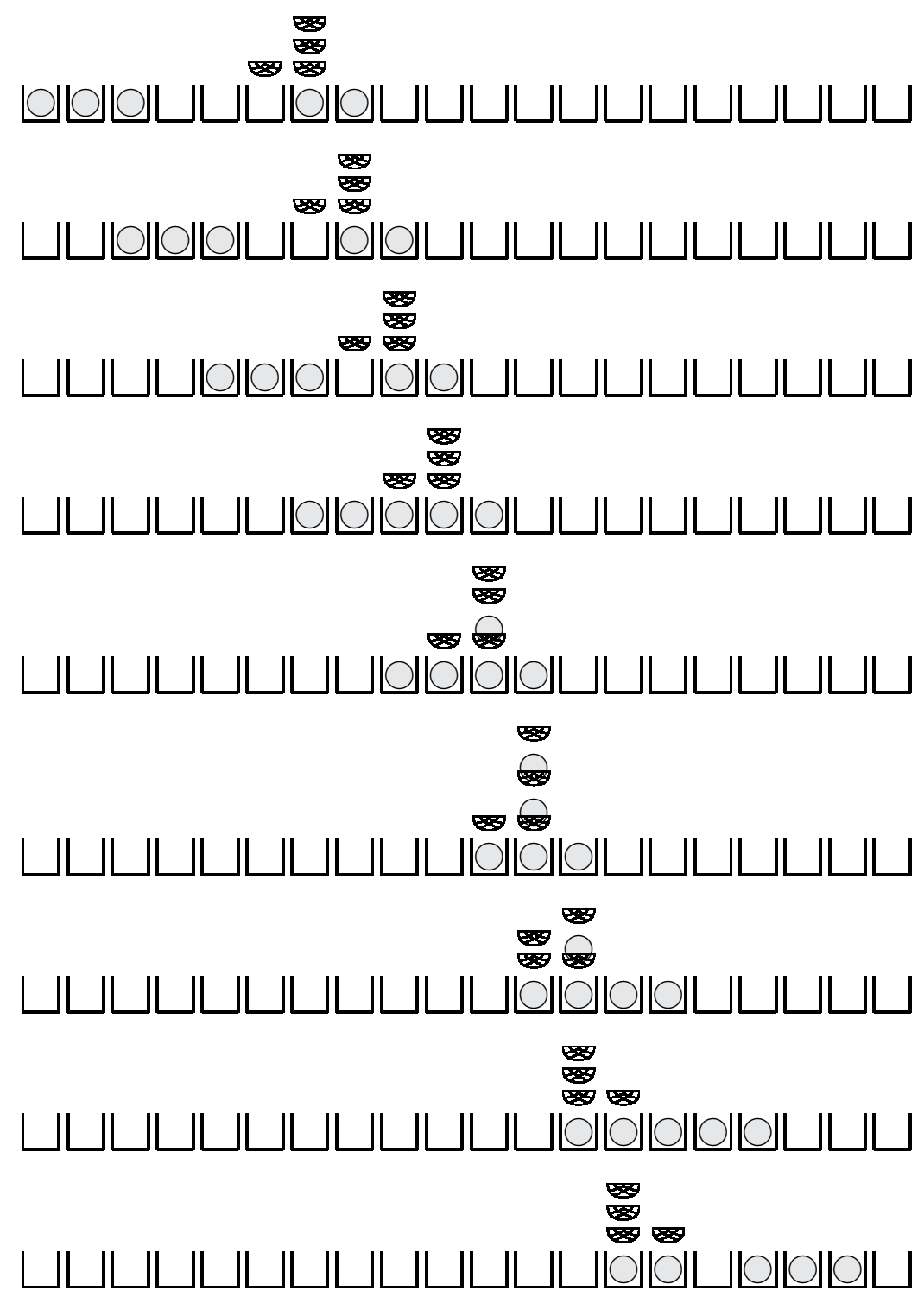

Figure 4. Scattering of $F_{3}$ and $B_{1} U_{3} F$ under $T_{2}$.

We can decompose composite basic solitons into disjoint union of pure solitons by multiple scatterings with $F_{k}$ 's. (By a disjoint union we mean that the basic solitions are separated by at least as many vacuum sites as the speed of the soliton.)

Proposition 5.5. For any time evolution $T_{\ell}(\ell \geq 2)$, consider multiple scattering of composite solitons with $F_{k}(k \geq 2)$ for sufficiently many times. Then the chunks described 
above decompose into disjoint union of pure solitons as follows:

$$
\begin{aligned}
& B_{a} U_{b_{1}} \ldots U_{b_{r}} \longmapsto F^{\sqcup r} \sqcup B_{a+b_{1}+\cdots b_{r}} \\
& B_{a} U_{b_{1}} \ldots U_{b_{r}} F \longmapsto F^{\sqcup r+1} \sqcup B_{a+b_{1}+\cdots b_{r}} \\
& V U_{b_{1}} \ldots U_{b_{r}} \longmapsto F^{\sqcup r} \sqcup B_{b_{1}+\cdots b_{r}} \\
& V U_{b_{1}} \ldots U_{b_{r}} F \longmapsto F^{\sqcup r+1} \sqcup B_{b_{1}+\cdots b_{r}} \\
& V F \longmapsto F \\
& B_{a_{1}, \cdots, a_{r}} \longmapsto B_{a_{1}, \cdots, a_{r}}
\end{aligned}
$$

Here $B_{0}=V$ and $F^{\sqcup r}:=\overbrace{F \sqcup F \sqcup \cdots \sqcup F}^{r}$.

Proof. The result essentially follows from Proposition 5.2, except that we must note that scattering of $F_{k}$ with $F \sqcup F \sqcup \cdots \sqcup F \sqcup A$ gives $F \sqcup F \sqcup \cdots \sqcup F \sqcup A^{\prime}$. In other words, the presence of the $F$ 's do not affect the scattering of $F_{k}$ with $A$. This is easy to establish by induction on the number of $F$ 's.

Given a disjoint union of basic solitons, we define the number of ball solitons and number of basket solitons as follows. For pure solitons, we consider $F_{k}$ as one ball soliton, whereas $B_{a_{1}, \cdots, a_{r}}$ is $r$ basket solitons. For a composite basic soliton states, we consider the decomposition in Proposition 5.5 and sum over the disjoint union of pure solitons. For a disjoint union of basic solitons, we sum over each basic soliton. We also define amplitudes of the resulting pure solitons as follows; amplitude of $F_{k}$ is $k$ and that of $B_{a}$ is $a$.

Remark 5.6. Alternatively, we may define the number of solitons of an arbitrary state by the total number of pure solitons obtained by making enough many scatterings of the state with ball solitons $F_{k}$ ( $k$ : large enough).

Example 5.7. Scatterings of more than $60 F_{k}(k \geq 2)$ with $U_{10} B_{7} B_{8} U_{12} U_{9} F B_{9} F$ under $T_{\ell}(\ell \geq 2)$ will give $F \sqcup F \sqcup F \sqcup F \sqcup F \sqcup B_{10,7,29,9}$. Here the original composite state is cut into chunks as $\left(U_{10}\right)\left(B_{7}\right)\left(B_{8} U_{12} U_{9} F\right)\left(B_{9}\right)(F)$ and the above proposition applies to each chunk. Thus the original state contains 5 ball solitons and 4 basket solitons.

By a $n$-scattering of solitons we mean the (large) time evolution of a disjoint union $\cdots A_{1} \cdots A_{2} \cdots \cdots A_{n} \cdots$ of $n$ solitions $A_{1}, A_{2}, \ldots, A_{n}$ such that the solitons are arranged in decreasing speed from left to right.

Theorem 5.8. The box-basket-ball system is solitonic in the following sense:

(1) from any initial state the system eventually evolves into disjoint union of basic solitons,

(2) the number and amplitudes of ball solitons and basket solitons contained in the initial state (after decomposition into pure solitons) are preserved under the time evolutions,

(3) the scattering of $n$ solitons is factorized into two body scatterings.

Proof. Consider the time evolution of the unbasketing of the system. By Theorem 2.1 at time $+\infty$ the state consists of separate solitons arranged in the order of non-decreasing speed. The baskets cannot move with speed bigger than one. Therefore after long enough time period all solitons in the unbasketing with speed greater than one shall come from solitons $F_{k}$ in the original box-basket-ball system. The unbasketed solitons of speed one 
come from some combination of states $V, F, B_{a}$ and $U_{a}$ avoiding subsequences $F F$ and $F U$. Thus they come from a union of basic speed one solitons in the box-basket-ball system.

For (2), let us consider a state $S$ which we assume is a disjoint union of basic solitons, arranged in decreasing order of speed. In order to count the number of solitons, we consider a state $S^{\prime}:=\left(F_{k} \otimes V^{\otimes k}\right)^{\otimes M} \otimes V^{\otimes L} \otimes S$ where $M$ is an integer as large as the decomposition in Proposition 5.5, and $L$ is a much larger integer than $M$. Then for a sufficiently large integer $N$, we can count the number of solitons from $T_{\infty}^{N}\left(S^{\prime}\right)$. We may assume that $k$ is large enough, and that the spacing in $S$ is large enough that there is no scattering between the original basic solitons in the calculation of $T_{\infty}^{N}\left(S^{\prime}\right)$.

Now let us consider the state $T_{\ell}^{K}\left(S^{\prime}\right)$, where $K$ is large enough that the scattering between basic solitons in $S$ is completed, while $\ell$ is chosen small enough (relative to $k$ and $L$ ) that the $F_{k}$ have not yet scattered with $S$. The number of solitons in the scattering of $S$ can then be counted by considering $T_{\infty}^{N} T_{\ell}^{K}\left(S^{\prime}\right)=T_{\ell}^{K} T_{\infty}^{N}\left(S^{\prime}\right)$ (see Theorem 4.4). Finally, $T_{\ell}^{K} T_{\infty}^{N}\left(S^{\prime}\right)$ is obtained from $T_{\infty}^{N}\left(S^{\prime}\right)$ by the scattering of some pure solitons. But by Proposition 5.2 this preserves the number of ball solitons and basket solitons. The preservation of amplitudes also follows from this argument.

The proof of (3) is exactly the same as that of [FOY, Theorem 4.6], and uses Theorem 4.4 and Proposition 4.3, we first evolve with $T_{2}$, which restricts to scatterings between speed $k>1$ and speed 1 solitons, then we evolve with $T_{3}$, and so on. After evolving with $T_{2}$, all the speed 1 solitons have been overtaken, so that when we evolve with $T_{3}$, we will restrict ourselves to scattering of speed $k>2$ and speed 2 solitons, and so on.

This characterization of the solitonic property is defined and proved for the box-ball system with capacity one boxes in [TNS]. Here we follow the treatment of [FOY]. For more general box-ball systems with boxes of arbitrary capacities, it is proved in [S1].

\section{Proof of FAST-SLOW SCATTERING FOR $T_{\infty}$}

Let us break up time evolution into

(A) Move baskets

(B) Move balls

(C) Reconfigure balls and baskets at each site separately

Each time step will correspond to applying $(\mathrm{A}),(\mathrm{B}),(\mathrm{C})$ in that order. So that "at time $t$ " we will have just completed step (C). To emphasize this we sometimes say "at integral time $t "$.

We shall adhere to the following rules when performing the moves $(\mathrm{B})$ and $(\mathrm{C})$. In move (B), if a fast ball is placed inside a special basket, it is designated a slow ball and the slow ball originally paired with the special basket is now called a fast ball. After the switch, we assume that the special basket and the new slow ball (in the special basket before (C)) are paired. In moves (B) or (C), we place balls in boxes first, then lower numbered baskets first, and in move $(\mathrm{C})$ if we have a choice, we shall place the slow ball in the box.

6.1. In the middle of a scattering. We shall first assume that no initial slow ball is present.

Lemma 6.1. There exist integers $t_{0}, t_{f}$ and integers $i_{t}, j_{t}$ for $t \in\left[t_{0}, t_{f}\right]$ so that (1) $1=i_{t_{0}} \leq j_{t_{0}}<i_{t_{0}+1} \leq j_{t_{0}+1}<\cdots<i_{t_{f}} \leq j_{t_{f}}=b$. 
(2) At time $t \in\left[t_{0}, t_{f}\right]$ the fast balls are located inside either boxes or the non-special baskets with numbers inside $\left[i_{t}, j_{t}\right]$.

(3) At times $t<t_{0}$, no interaction between the solitons has occurred. At times $t>t_{f}$, the scattering is complete and the output fast soliton is of the same length as before.

\section{Lemma 6.2.}

(1) Special baskets are always empty at integral time $t$. Thus they do not experience phase shift.

(2) Non-special baskets are occupied at exactly one integral time. Thus they are phase shifted by -1 .

(3) Non-initial slow balls experience a phase shift of -1 .

Proof. We shall establish Lemma 6.1 and 6.2 by induction.

We say that a special basket is activated when a fast ball is placed inside it during move (B). We first remark that assuming Lemma 6.2(1), we can show that at integral times a special basket is always one site behind its paired slow ball before activation, and at the same site as its paired slow ball after activation. Since when it is activated, the paired slow ball is the same site with the paired special basket after (A). Then the slow ball is interchanged with the fast ball in the spacial basket when activation occurs. Thus the slow ball enters the special basket and will not be moved by the rest of the move (B) so that it stays at the same site with the paired special basket.

We show Lemma 6.1 (1,2) for the first time $t=t_{0}$. At time $t=t_{0}-1+(A)$, any special baskets have been moved to the same site as the paired slow ball. At $t=t_{0}-1+(A, B)$, this special basket may or may not be occupied, but after move $(\mathrm{C})$, it will be unoccupied. (The original paired slow ball will be moved, and during move $(\mathrm{C}$ ) the special basket will be emptied.) Here the move (B) has the following property; once the first fast ball is moved, then no slow balls will be moved until all fast balls are moved. Assuming there is no initial slow ball, all slow balls are paired with special baskets. Then if a fast ball is put into a special basket, it is immediately interchanged by the paired slow ball and the new fast ball will be moved by the rest of $(B)$.

Now suppose the description of Lemma 6.1 (1,2) holds at time $t$ and consider the next step. In move $(\mathrm{A})$, all except the non-special baskets in $\left[i_{t}, j_{t}\right]$ will be moved towards the right. Then after the move $(\mathrm{A})$, non-activated special baskets are at the same site as the paired slow ball. The already activated special baskets are one site ahead of the paired slow ball. Note that we may assume by possibly changing the $j_{t}$ by 1 that any special baskets numbered in $\left[1, j_{t}\right]$ have been activated.

In move (B), the balls will move according to the following four steps:

(1) All slow balls to the left of any fast balls will move to the same site as their activated special baskets.

(2) The first $k$ fast balls, where $k$ is the number of activated special baskets numbered in the region $\left[i_{t}, j_{t}\right]$ will move into activated special baskets and immediately renamed as slow balls.

(3) The remainder of the fast balls, including any originally slow balls (now renamed as fast) which are paired with the activated special baskets in the second step or with newly activated special baskets in the third step will begin to fill up baskets labeled $j_{t}+1, j_{t}+2, \ldots$ and so on.

(4) The rest of the balls, all of which are slow will move one step forward into a box. 
Just as in the time $t_{0}$ we can show that no slow balls are touched during steps 2 and 3 . Thus the above four steps give the complete classification of the move (B) except for the following cases. In the final step it is possible for a slow ball to end up in a basket rather than box; and in the earlier steps it is possible for balls to end up in boxes rather than baskets. These exceptions do not affect the result of Lemma 6.1 and 6.2,

At time (C), every occupied special basket which has been activated will now be emptied - the original paired slow ball (now designated a fast ball) has left the site, and the slow ball inside the special basket can be placed into the box. This shows that Lemma 6.1(1,2) holds at all times. Lemma 6.1(3) also holds since the number of fast balls remain constant, and they will come out all consecutive.

Lemma 6.2 $(1,2,3)$ all follow from the above discussion. For (3), one just notes that the slow ball is always one ahead (at integral time) of the paired special basket before activation, and always in the same site after activation.

6.2. Initial slow ball. The analysis of the system containing an initial slow ball can be reduced to that of no initial slow ball case. The behaviour is just the same as the (known) scattering of $F_{k}$ and $F_{1}$.

Lemma 6.3. Any initial slow ball is phase shifted by -2, but does not affect the rest of the scattering.

Proof. When the initial slow ball is first overtaken by a fast ball, say at time $t+(A, B)$, we rename the left-most fast ball a slow ball (and now consider the initial slow ball a fast ball). At time $t+2$, the new slow ball will be in the location of the original slow ball at time $t$. This gives the calculation of the phase shift. The rest of the scattering then proceeds in the original manner.

\subsection{Phase shift of fast soliton.}

Lemma 6.4. Suppose there are a baskets and b balls in the slow soliton. Then after scattering, the fast soliton is phase shifted by $2 b-a$.

Proof. Every time a fast ball is placed into a non-special basket, the fast soliton is slowed by 1 unit. When a special basket is first activated, the fast ball becomes a slow ball, and a new fast ball replaces the old one at the same site. Since the new fast ball is occupying a box, this does not change the speed of the soliton, except that the new fast ball has yet to move. And when the new fast ball moves, the fast soliton is sped up by 1 unit. The next time the activated special basket is occupied, there is no effect on the speed of the fast balls (the special basket traps a fast ball, but then a new fast ball will replace it).

Acknowledgments. T.L. is supported by NSF grant DMS-0901111, and by a Sloan Fellowship. P.P. was supported by NSF grant DMS-0757165. R.S. is partially supported by Grants-in-Aid for Scientific Research No. 21740114 from JSPS.

\section{REFERENCES}

[BKK] G. Benkart, S.-J. Kang And M. Kashiwara: Crystal bases for the quantum superalgebra $U_{q}(\mathfrak{g l}(m, n))$. J. Amer. Math. Soc. 13 (2000), no. 2, 295-331.

[DS] L. DEKA And A. Schilling: New fermionic formula for unrestricted Kostka polynomials. J. Combinatorial Theory, Series A 113 (2006) 1435-1461

[FOY] K. Fukuda, M. OKado And Y. Yamada: Energy functions in box ball systems. Internat. J. Modern Phys. A 15 (2000), no. 9, 1379-1392. 
[HhikTt] G. Hatayama, K. Hikami, R. Inoue, A. Kuniba, T. Takagi and T. Tokihiro: The $A_{M}^{(1)}$ automata related to crystals of symmetric tensors. J. Math. Phys. 42 (2001), no. 1, 274-308.

[HI] K. Hikami And R. Inoue: Supersymmetric extension of the integrable box-ball system. J. Phys. A: Math. Gen. 33 (2000) 4081-4094.

[K] M. Kashiwara: On crystal bases of the $q$-analogue of universal enveloping algebras. Duke Math. J. 63 (1991), no. 2, 465-516.

[KKR] S. V. Kerov, A. N. Kirillov and N. Yu. Reshetikhin: Combinatorics, the Bethe ansatz and representations of the symmetric group. Zap. Nauchn. Sem. (LOMI) 155 (1986) 50-64. (English translation: J. Sov. Math. 41 (1988) 916-924.)

[KOSTY] A. Kuniba, M. Okado, R. Sakamoto, T. Takagi and Y. Yamada: Crystal interpretation of Kerov-Kirillov-Reshetikhin bijection. Nuclear Phys. B 740 (2006), no. 3, 299-327.

[KSY] A. Kuniba, R. Sakamoto, and Y. Yamada: Tau functions in combinatorial Bethe ansatz. Nuclear Phys. B 786 (2007), no. 3, 207-266.

[LP] T. Lam and P. Pylyavskyy: Crystals and total positivity on orientable surfaces, preprint, 2010; arXiv: 1008.1949 .

[OSS] M. Okado, R. Sakamoto And A. Schilling: Affine crystal structure on rigged configurations of type $D_{n}^{(1)}$, preprint, 2011; arXiv:1109.3523,

[PST] J. K. Park, K. Steiglitz and W. P. Thurston: Soliton-like behavior in automata. Physica D: Nonlinear Phenomena 19 (1986), 423-432.

[S1] R. Sakamoto: Crystal interpretation of Kerov-Kirillov-Reshetikhin bijection. II. Proof for $\mathfrak{s l}_{n}$ case. J. Algebraic Combin. 27 (2008), no. 1, 55-98.

[S2] R. Sakamoto: Kirillov-Schilling-Shimozono bijection as energy functions of crystals. Int. Math. Res. Not. IMRN 2009, no. 4, 579-614.

[S3] A. Schilling: Crystal structure on rigged configurations. Int. Math. Res. Not. IMRN 2006, Article ID 97376, Pages 1-27.

[S4] A. Schilling: Combinatorial structure of Kirillov-Reshetikhin crystals of type $D_{n}^{(1)}, B_{n}^{(1)}, A_{2 n-1}^{(2)}$. J. Algebra 319 (2008) 2938-2962.

[T] D. TAkahashi: On some soliton systems defined by using boxes and balls, Proceedings of the International Symposium on Nonlinear Theory and Its Applications (NOLTA '93), (1993) 555-558.

[TM] D. TAKahashi and J. Matsukidaira: Box and ball system with a carrier and ultradiscrete modified KdV equation. J. Phys. A 30 (1997), no. 21, L733-L739.

[TS] D. Takahashi And J. Satsuma: A soliton cellular automaton. J. Phys. Soc. Japan 59 (1990), no. $10,3514-3519$.

[TNS] T. Tokiniro, A. Nagai and J. Satsuma: Proof of solitonical nature of box and ball systems by means of inverse ultra-discretization. Inverse Problems 15 (1999), no. 6, 1639-1662.

[TTMS] T. Tokiniro, D. Takahashi, J. Matsukidaira and J. Satsuma: From soliton equations to integrable cellular automata through a limiting procedure. Phys. Rev. Lett. 76 (1996) 3247-3250.

E-mail address: tfylam@umich.edu

Department of Mathematics, University of Michigan, 530 Church St., Ann Arbor, Mi 48109 USA

E-mail address: ppylyavs@math.umn.edu

Department of Mathematics, University of Minnesota, 206 Church St. Se, MinneapoLIS, MN 55455 USA

E-mail address: reiho@rs.tus.ac.jp

Department of Physics, Tokyo University of Science, Kagurazaka, Shinjukuku, Tokyo 162-8601 JAPAN 